\title{
Téoros
}

Revue de recherche en tourisme

\section{Un numéro qui a fait jaser}

Volume 4, numéro 2, juillet 1985

URI : https://id.erudit.org/iderudit/1080947ar

DOI : https://doi.org/10.7202/1080947ar

Aller au sommaire du numéro

Éditeur(s)

Université du Québec à Montréal

ISSN

0712-8657 (imprimé)

1923-2705 (numérique)

Découvrir la revue

Citer ce document

(1985). Un numéro qui a fait jaser. Téoros, 4(2), 37-37.

https://doi.org/10.7202/1080947ar

Ce document est protégé par la loi sur le droit d'auteur. L'utilisation des services d'Érudit (y compris la reproduction) est assujettie à sa politique d'utilisation que vous pouvez consulter en ligne.

https://apropos.erudit.org/fr/usagers/politique-dutilisation/
Cet article est diffusé et préservé par Érudit.

Érudit est un consortium interuniversitaire sans but lucratif composé de l’Université de Montréal, l'Université Laval et l'Université du Québec à Montréal. Il a pour mission la promotion et la valorisation de la recherche. https://www.erudit.org/fr/ 


\section{Un numéro qui a fait jaser}

Le numẹro précédent consacré à l'information touristique (vol 4 , no. 1) a suscité plusieurs réactions. Plusieurs de nos lecteurs nous ont fait connaitre leur satisfaction par rapport au thème du numéro et par rapport à la qualité des articles et a leur présentation. D'autres lecteurs ne partageaient pas tout a fait les points de vue des auteurs des articles sur tel ou tel aspect abordé par eux tandis que certains ont tenu à nous souligner quelques oublis. Mais, il est bon de se le rappeler, ce numero n'entendait pas atre exhaustif sur toutes les questions reliées a P'information touristique. II s'agissait, comme le soulignait Normand Cazelais dans la presențation du numéro, de donner la parole aux artisans de l'information, le numéro étant constitue de témoignages, de visions de /interieur.

Voici deux lettres qui rêvèlent l'intérêt que l'on a porté á ce numéro et nous remercions les auteurs de ces lettres de nous avoir fait parvenir leurs commentaires écrits.

Louis Jolin

Directeur

La Revue Téoros

\section{L'information touristique à La Presse}

Monsieur le Directeur.

C'est avec plaisir que j’ai lu le numéro de mars 1985 que votre revue a consacre à l'information touristique au Québec et, en particulier, au journal La Presse.

La diwersité des articles, la qualité de certains témoignages et l'excellent travail de coordination de M. Normand Cazelais ont contribue a rendre ce numero de Téoros fort enrichissant.

Les résultats de l'enquẹte réalisée sous la direction de M. Jean Stafford auprés des lecteurs du Cahier Vacances-Vovage de La Presse démontrent, sans jeu de mots, que nous ne faisons pas fausse route.

Je me permets toutefois de répondre à l'article de Pierre Vincent, intitulé $D u$ Réve a la réalité, sur l'information touristique a La Presse en apportant, pour le benéfice de vos lecteurs, les précisions suivantes :

1.- Les thèmes du cahier Vacances-Voyage sont fixés par la Direction de l'Information, en collaboration avec le responsable de ce cahier, M. Michel-G. Tremblay, en tenant compte de l'actualité Isaisons, nouvelles destinations, etc.) et des besoins de nos lecteurs lles destinations exotiques ne nous font pas oublier le Canada ou les Etats-Unis, beaucoup plus accessibles).
2.- De janvier 1983 à avril 1965 , les themes du cahier ont été les suivants :

* 30 cahiers sur les Etats-Unis (25\%)

- 42 cahiers sur tous les pays d'Europe (35\%)

- 9 cahiers sur les destinations canadiennes $(7 \%)$

- 12 cahiers sur les Antilles et le Mexique $(10 \%)$

- 3 cahiers sur I'Asie $(2 \%)$

- 3 cahiers sur l'Afrique et le Moyen-Orient (2\%)

- 4 cahiers sur l'Amérique du Sud $(2 \%)$

- 15 pages-cahiers sur le Québec $(13 \%)$ plus 30 chroniques hebdomadaires de Lilianne Lacroix qui a obtenu en 1983 le prix du jour. nalisme de Loisirs au Québec pour cette chronique publiée dans le cahier.

3.- La majorité des articles sont rédigés par nos chroniqueurs touristiques, selon des criteres professionnels. Le lecteur $y$ trouve ainsi une garantie de sérieux. Nous ne croyons pas a la formule selon laquelle plus nous serions de fous a écrire, mieux tout le monde serait informe. Le chroniqueur au tourisme est un spécialiste au mẻme titre que le chroniqueur de cinéma ou de hockey.

4." Nous ne demandons pas à nos chroniqueurs au tourisme de toujouss être positifs ou de ghisser sur les aspects négatifs d"une destination de vacances. Par exemple, un reportage sur Haïti devra comprendre des données évidentes : régime politique, pauvreté des habitants, qualité de l'eau, faiblesse du réseau routier. Devrons nous tairé pour autant la richesse historique du pays, la chaleur des Haitiens, lanimation de ses villages, les prix abordables? Le lecteur jugera. II importe, pour nous, de né pas le tromper et de nous souvenir qu'il lit des repontages qui doivent liaider as planifier ses vacances. Les reportages sociopolitiques trouvent leur place ailleurs dans te journal.

5.- Si nous acceptons, comme tous les médias d'information, des invitations de gros. sistes ou de transporteurs, ils sont toujours prévenus, au départ, que nous gardonsi notre entière liberté professionnelle. Sans jouer aux puristes, cette formule permet de faire connailtre â nos lecteurs des destinations auxquelles, pour des raisons budgétaires ${ }^{\text {enous }}$ devrións renoncer.

6.- La Presse alloue a ses chroniqueurs au tourisme un budget de voyage annuel $1 \$ 40,000$ en 1985) leur permettant de visiter des pays ou de compléter des itineraires susceptibles d'intéresser nos lecteurs.

7.. Nous ne sommes pas à l'abri des pressions des transporteurs, des grossistes, des pays ou des agences de publicite. Non seulement ces pressions ne sont-elles pas plus fortes que dans d'autres secteurs d'activitės, mais La Presse, avec un tirage de 320,000 exemplaires et plus d'un million de lecteurs le samedi, peut dèterminer elle-mème ses priorités.

8.- Dans le monde de linformation touristique, La Presse s'est taillée au fils des ans, gráce a la qualité professionnelle de ses reportages et a l'excellence de ses journalistes, une réputation et une crédibilité dont nous sommes fiers. Laisser croire que nous cédons volontiers aux pressions des vendeurs de rëves relève de la pure fantaisie. Et pour une raison tres simple, qui tient au bon sens : tromper la confiance de nos lecteurs reviendrait à nous détruire.

Je vous pris d'agréer, Monsieur le Directeur, l'expression de mes sentiments les meilleurs.

Le Directeur de l'Information,

Claude Gravel

\section{Un oubli malencontreux}

\begin{abstract}
Monsieur Jolin,
Depuis plusieurs annés, l'organisme que je représente, l'Organisation pour le tourisme étudiant au Québec, s'occupe à promouvoir le voyage en distribuant de l'information aux étudiants de niveau post-secondaire. Croyant fermement a notre mission d'éducation, wous pouvez vous imaginer ma joie lorsque jappris le thème de votre numéro de février. Enfin quelqu' un reconnaissait l'importance de l'information touristique! Bravo pour cette initiative.
\end{abstract}

Malheureusement, en dévorant la revue je n'ai vu aucune mention concernant les 38 bureaux d'information voyages des cégeps et universités. Ceux-ci. opérés par 500 bẹnévoles, ont repondu a près de 40,000 demandes d'information en 198384. Pour l'année qui vien de se terminer, l'impact semble encore plus fort. Une telle initiative aurait dô, selon nous, ẗtre mentionnée.

De plus, I'OTEO publie aussi un magazine: Temps libre. Distribué gratuitement 6 fois par annee, Temps libre rejoint plus de 100,000 personnes par parution grace a ses 50,000 copies. Malgré son jeune agge (2 ans), nous crovons aussi qu'il aurait do être mentionne comme outil d'information important, principalement pour les jeunes.

Nous avons toujours apprécies votre revue et nous vous encourageons a continuer puisqu'elle est primordiale pour le développement du tourisme québécois. Nous estimons toutefois que le numéro sur l'information touristique manquait peut-être d'un peus de recherches..

Bien à wous,

Robert Bisson,

Président 